\title{
Automatically countering imbalance and its empirical relationship to cost
}

\author{
Nitesh V. Chawla \\ University of Notre Dame \\ Notre Dame, IN 46556, USA \\ David A. Cieslak \\ University of Notre Dame \\ Notre Dame, IN 46556, USA \\ Lawrence O. Hall \\ University of South Florida \\ Tampa, FL 33620-5399, USA
}

NCHAWLA@CSE.ND.EDU

HALL@CSE.USF.EDU

Ajay Joshi

AJOSHI@CSE.USF.EDU

University of South Florida

Tampa, FL 33620-5399, USA

\section{Editor:}

\begin{abstract}
Learning from imbalanced datasets presents a convoluted problem both from the modeling and cost standpoints. In particular, when a class is of great interest but occurs relatively rarely such as in cases of fraud, instances of disease, and regions of interest in largescale simulations, there is a correspondingly high cost for the misclassification of rare events. Under such circumstances, the data set is often re-sampled to generate models with high minority class accuracy. However, the sampling methods face a common, but important, criticism: how to automatically discover the amount and type of sampling? To address this problem, we propose a wrapper paradigm that discovers the amount of resampling for a data set based on optimizing evaluation functions like the f-measure, Area Under the ROC Curve (AUROC), cost, cost-curves, and cost dependent f-measure. Our analysis of the wrapper is two-fold. First, we report the interaction between different evaluation and wrapper optimization functions. Secondly, we present a set of results in a cost-sensitive environment, including scenarios of unknown or changing cost matrices. We also compare the performance of the wrapper approach versus cost-sensitive learning methods - MetaCost and Cost-Sensitive Classifiers - and find the wrapper to out-perform cost-sensitive classifiers in a cost-sensitive environment. Lastly, we obtain the lowest cost per test example compared to any result we are aware of for the KDD-99 Cup intrusion detection data set.
\end{abstract}

\section{Introduction}

Imbalance in class distribution is pervasive in a variety of real-world applications, including but not limited to telecommunications, WWW, finance, biology and medicine. The minority or positive class is often of interest and also accompanied with a higher cost of making errors. A typical example of this problem is fraud detection. The instances of fraud in 
the population are generally a very small proportion (often in the neighborhood of $2 \%$ ). However, it is quite important to be able to detect a fraudulent transaction. At the same time, it is also important to minimize false positives because these result in investigation cost and can also result in losing a customer. Thus, there is a distribution of costs associated with both false positives and false negatives. Another example is large-scale simulation. While, there are not "dollar" costs attached with errors like there may be with fraud detection, there is a cost attached to time spent in poring over "uninteresting regions" in a simulation. It is known that the regions of interest in such simulations are typically rare (Bowyer et al. (2000); Banfield et al. (2005)). Hence, an intelligent tool should be accurate in identifying interesting regions, without too many false alarms.

Weiss and Provost (2003) observed that the naturally occurring distribution is not always optimal. Thus, one needs to modify the data distribution, conditioned on an evaluation function. Re-sampling, by inflating the minority (positive) class or deflating the majority (negative) class of a given data set, has become a de facto standard to counter the curse of imbalance in various domains. There have been numerous papers and case studies exemplifying their advantages (Chawla et al. (2004); Kubat and Matwin (1997); Ling and Li (1998); Weiss and Provost (2003); Ferri et al. (2004); Dietterich et al. (2003); Kubat et al. (1998a); Chawla et al. (2003); Zadrozny and Elkan (2001); Batista et al. (2004); Maloof (2003); Drummond and Holte (2003)). However, the one common critique of various works is: how does one effectively identify the potentially optimal sampling technique and parameters for a given data set? An accompanying question is: can the techniques for imbalance generalize across cost-sensitive scenarios?

Driven by these important and pertinent questions and appreciative of the problem that sampling will be highly data dependent, we propose a wrapper framework for empirically discovering the sampling parameters. We fill an important hole in the application of sampling methods to counter the problem of class imbalance by comprehensively demonstrating the efficacy of wrapper paradigm. Our goal was to empirically evaluate the usefulness of popular techniques for countering class imbalance by tuning the parameters on a validation set before reporting the performance on the testing set. All the work, thus far, has reported the best performance on the testing set by applying different sampling strategies. Thus, there is no empirical estimate of generalization capacity. In addition, we evaluate the generalization performance of the classifiers, learned on such re-sampled datasets, in a large variety of experimental scenarios. We want to analyze the flexibility of learned models in a dynamic environment since misclassification costs are often unknown (Weiss et al. (2007)) or may change. We wanted to empirically investigate the relationship between choosing the "best" sampling strategy via a wrapper method and its relationship to classification in a cost-sensitive environment, especially when the costs are unknown or can change. Thus, the over-arching question is: Can we utilize measures and methods that counter class imbalance and do well in a cost-sensitive framework?

Contributions Our main contributions in this paper are centered around the following research questions.

- Can we effectively discover sampling strategies for a given classifier and domain? 
- Does the proposed wrapper paradigm for countering imbalance, guided by cost-insensitive criteria such as the area under the receiver operator characteristic curve $A U R O C$ and the $f$-measure, perform well in a cost-sensitive testing environment? How does it compare to using cost directly in the wrapper mode to guide the search? How do changing costs or unknown costs effect the performance of the wrapper?

- In a cost-sensitive environment, are there potential advantages in accounting for the imbalance first by applying sampling strategies as compared to MetaCost (Domingos (1999)) and Cost-Sensitive Classifier (Zadrozny et al. (2003b))?

The remainder of this paper is organized as follows. Section 2 introduces the wrapper framework to discover the coupling of a sampling method and corresponding parameters with a classifier and domain. Section 3 presents the experimental framework. Our empirical analyses first illustrate the efficacy of the wrapper framework across datasets and using cost-transparent evaluation measures in Section 4. Then in Section 5, the impact of introducing costs during testing is demonstrated using a variety of cost-ratios. Finally, Section 6 discusses the results.

\section{Wrapper Paradigm}

Re-sampling is a popular solution to the class imbalance problem. However, one persistent limitation of the sampling methods has been automatically discovering the amount and type of sampling to apply given a particular data set. To address this limitation, we propose a comprehensive wrapper infrastructure that applies cross-validation to first discover the best amounts of undersampling and oversampling. For oversampling, we use the Synthetic Minority Over-Sampling TEchnique (SMOTE) (Chawla et al. (2002)), which generates synthetic examples by selecting new examples in random intervals between positive class nearest neighbors. The new examples are then added to existing minority class examples. Obviously, searching the entire space of undersampling and SMOTE combinations can quickly become intractable, so we proceed in a step-wise fashion. This strategy removes the "excess" examples of the majority classes, reducing the size of the training data set and making learning time more tractable. Then SMOTE is used to add synthetic examples of the minority classes and increase the generalization performance of the classifier over the minority classes. Figure 1 shows the Wrapper_UnderSample_SMOTE algorithm, which can extend to multiple majority and minority classes. The metric values in the pseudo-code indicate the performance criterion, which will be discussed in subsequent sections. We will analyze the cross-validation approach used in Section 2.1, outline the wrapper approach to selecting sampling levels in Sections $2.2 \& 2.3$, and survey the wrapper search evaluation metrics in Section 2.4.

\subsection{Using Cross-Validation to Guide Sampling}

The wrapper approaches were optimized on an independent validation set to avoid any bias in performance on the testing data. Thus, we construct a careful cross-validation framework. We first split the data set into ten partitions. Each partition is used once as a testing fold, with the remaining $90 \%$ as the training fold. This results in ten pairs of 
training and testing folds (10-fold cross-validation), forming the basis for our comparison of the different methods. We further split each of the training folds, independently, in to five partitions for an internal 5-fold cross-validation. The wrapper applies this independent validation stage to each fold to discover the appropriate percentages of sampling for a given method and classifier combination. Once these percentages are discovered, the classifier is re-learned on the original training fold using the discovered percentages and tested on the corresponding testing fold. Thus, the purpose of internal 5-fold cross-validation is only to guide an independent wrapper stage, keeping the original testing fold separate for the honest assessment of the performance. Note that this procedure is repeated for each of the 10 -folds. Furthermore, due to the inherent random nature of undersampling and SMOTE, the process of training and testing with wrapper selected undersampling and SMOTE percentages was done a total of five times to get an averaged (more stable) performance measure.

Algorithm WRAPPER_UNDERSAMPLE_SMOTE(MinorList, MajorList, NumFolds)

1: Input: List of minority classes Minor List

2: List of majority classes Major List

3: Number of cross-validation folds NumFolds

4: Output: Wrapper selected UnderSampling percentages for majority classes; Wrapper selected SMOTE percentages for minority classes

5: for all MClass in MajorList do

6: $\quad$ UnderSampleList $[$ MClass $]=100$

7: end for

8: for all MClass in MinorList do

9: $\quad$ SmoteList $[$ MClass $]=0$

10: end for

11: for Fold $\leftarrow 1$ to NumFolds do

12: $\quad$ Build classifier on training fold Fold and evaluate on validation set

13: $\quad$ Update BestMinorMetricValues and BestMajorMetricValues

14: end for

15: if Major List is not empty then

16: WRAPPER_UNDERSAMPLE(UnderSampleList)

17: end if

18: if Minor List is not empty then

19: WRAPPER_SMOTE(SmoteList,UnderSampleList)

20: end if

21: Output UnderSampleList and (SmoteSampleList)

Figure 1: Wrapper Undersample SMOTE Algorithm

Lastly, the entire procedure was repeated five times to achieve more stable estimates in the performance. To summarize, for five different runs, the ten folds were constructed and on each fold a five-fold cross-validation was applied to discover the amounts of SMOTE and undersampling. The final reported performances are the averages over the 50 sets $(5$ runs $\times$ 10 folds). We will now explore the heuristic functions used to optimize the sampling levels. 


\subsection{Wrapper Based Algorithm to Select Undersampling Percentages}

This algorithm uses a wrapper approach to perform the search through the parameter space of undersampling percentages for the majority class(es), using the chosen learning algorithm as a part of the evaluation function for a five-fold cross-validation on the training data. The purpose is to search for the sampling level in the parameter space with the highest evaluation score guided by some heuristic function. This search in the parameter space has to be restricted to the training data to avoid any estimation biases. Thus, we utilized a five-fold cross-validation on the training set to discover the amount of undersampling.

The wrapper starts with no undersampling for all majority classes and obtains baseline results on the training data. Then it traverses through the search space of undersampling percentages in decrements of SampleDecrement (in this case 10\%), in a greedy iterative fashion, to increase performance over the minority classes without sacrificing performance on the majority class. We start with $100 \%$ undersampling, implying that all of the majority class examples are retained. And then we remove the majority class examples $10 \%$ at a time. This search process continues as long as it does not hamper the accuracy of any minority class (normally the accuracy of the minority classes increases) or drop the accuracy for any majority class more than some specified amount IncrementMin ( $5 \%$ for our experiments). The algorithm terminates when the performance threshold is violated for any class specified in MajorList or MinorList, to prevent performance deterioration for any class. The details are presented in Figure 2.

\subsection{Wrapper Based Algorithm to Select SMOTE Percentages}

The dataset was undersampled by the amounts discovered in the previous step. We chose to undersample first, as we wanted to first eliminate any majority class examples that did not add any improvement in the evaluation metric. Moreover, it also reduced the size of the training set, speeding up learning. Now the performance obtained from undersampling was the new benchmark or baseline. We only decide to SMOTE if it improves performance over the baseline. Now the Wrapper SMOTE algorithm continues to step through the search space for the SMOTE percentage of minority classes by SampleIncrement (in this case $100 \%$ ) in a greedy fashion and obtains new performance estimates using a five-fold crossvalidation over the training data. If the average metric value over the minority classes was improved by margin IncrementMin (typically 5\%; see line 17 in the algorithm), then the current results become the next baseline to beat, and the search continued to find better SMOTE percentage combinations for the minority classes.

If the average does not improve by $5 \%$ then to verify that we have not settled on a local maximum, we look ahead two more steps at increasing amounts of SMOTE. If the look-ahead does not result in an improvement in performance, then the amount of SMOTE is reset to the value discovered prior to the look-aheads. This is done to allow SMOTE to introduce additional examples with the aim of improving performance. However, if the addition of examples does not help, then we go back to using the lesser amount of SMOTE discovered prior to the look-ahead. The pseudo code for the wrapper SMOTE algorithm is presented in Figure 3.

We do not check for the Major MetricValue, as we are primarily interested in optimizing the performance on the minority class in this stage, with the premise that the true positives 


\section{Algorithm $W R A P P E R \_U N D E R S A M P L E(U n d e r S a m p l e L i s t)$}

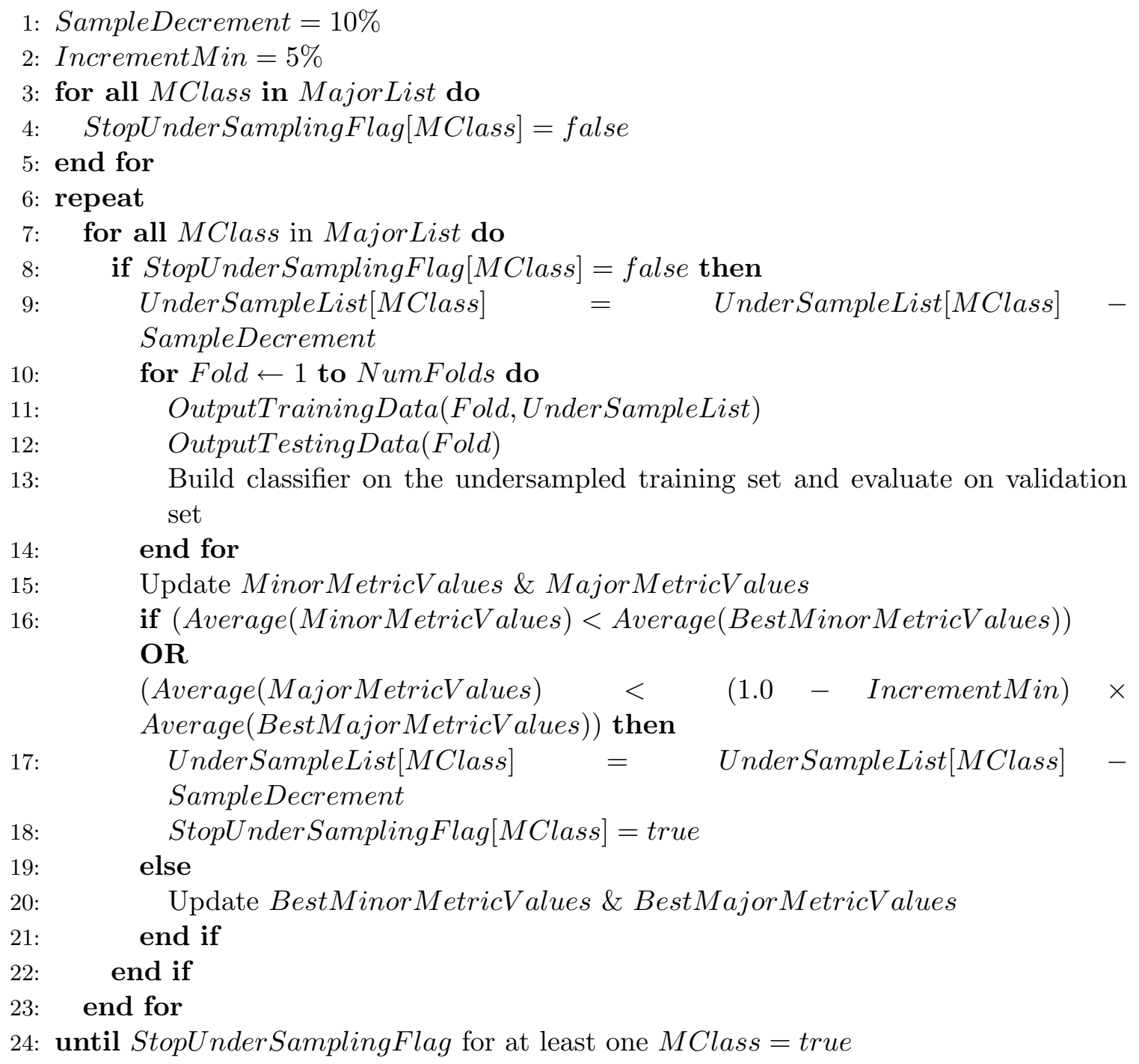

Figure 2: Wrapper Undersample Algorithm 
Algorithm WRAPPER_SMOTE(SmoteList,UnderSampleList)

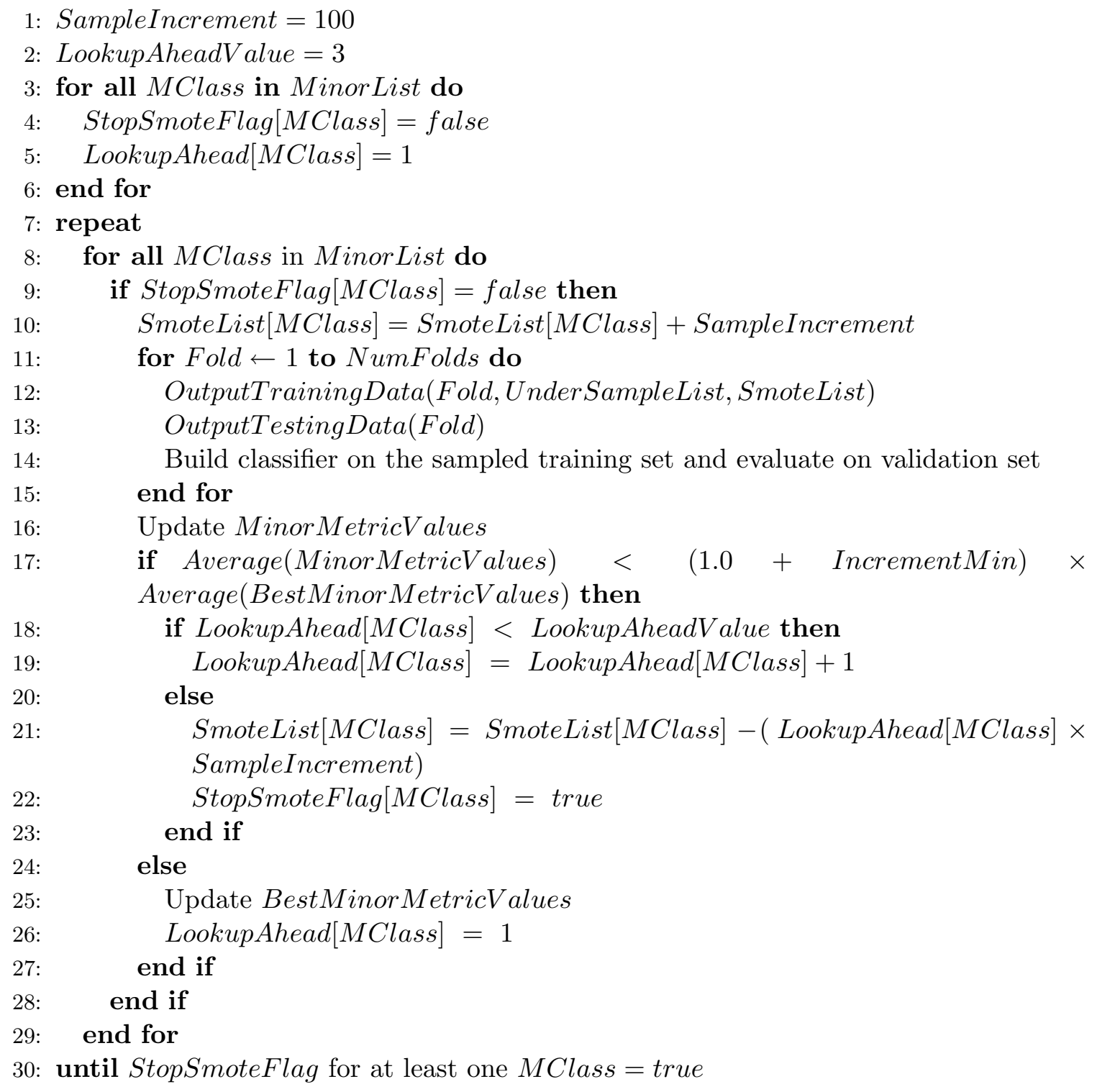

Figure 3: Wrapper SMOTE Algorithm 
are more important than false positives. We do not perform a look-ahead for undersampling since we believe that undersampling effectively reduces the information contained in the data and thereby impedes the classification performance. The chief purpose of undersampling is to remove redundant majority class examples without reducing the overall accuracy of the classifier.

\subsection{Evaluation Metrics}

The only way to determine optimal sampling levels is through empirical analysis. Crossvalidation is required because optimality of the sampling methods must be determined on the training data only. One question remains: given our sampling space, how does one determine the "best" sampling levels? Thus, we will explore four classifier evaluation criteria which were deployed and empirically tested.

\subsubsection{Cost}

When optimizing sampling levels to improve overall cost, a logical evaluation criteria is the cost itself. Cost is calculated as shown in Equation 1 when we assume $C(+\mid+)=C(-\mid-)=$ 0 .

$$
\text { cost }=\text { FNrate } \times C(-\mid+)+\text { FPrate } \times C(+\mid-)
$$

Therefore, once classification occurs in the cross-validation phase, the wrapper calculates the validation cost and uses this information to select optimal sampling levels. This approach is dependent on a priori knowledge of the cost relationship between classes. Different cost ratios may cause selection of the different optimal sampling levels and thus generate very different classifiers.

In the subsequent discussion, we will refer to the wrapper approach using cost as wrappercost.

\subsubsection{F-MEASURE}

The $f$-measure tends to be used in information retrieval. It is a composite metric based on precision and recall (Dumais et al. (1998); Mladenic and Grobelnik (1999); Lewis and Ringuette (1994); Cohen (1995b)), where precision and recall are defined as follows:

$$
\begin{gathered}
\text { precision }=\frac{T P}{T P+F P} \\
\text { recall }=\frac{T P}{T P+F N}
\end{gathered}
$$

As a composite statistic, $f$-measure is then calculated from precision and recall to summarize the effects of the two types of errors.

$$
\text { fmeasure }=\frac{2 \times \text { precision } \times \text { recall }}{\text { recall }+ \text { precision }}
$$

It is desirable to increase recall without a sacrifice in precision. Therefore, an effective wrapper strategy is to determine the sampling levels which maximize the classifier's $f$ measure. This reduces error on the relatively expensive minority class while maintaining 
accuracy on the majority class. This wrapper evaluation function has an advantage over the cost-based wrapper in that a priori knowledge of the cost matrices is not required. The generated classifier can be consistently applicable throughout alternative unseen class distributions and cost relationships. In the subsequent discussion, we refer to the wrapper approach using the f-measure as wrapper-fmeasure.

\section{$2.4 .3 \beta$ VARIED $f$-measure}

Another wrapper evaluation criterion is based on an alternate derivation of the previously outlined $f$-measure. Here, a separate $\beta$ factor is incorporated to introduce an element of relative importance between recall and precision:

$$
\text { fmeasure }_{\beta}=\frac{\left(1+\beta^{2}\right) \times \text { precision } \times \text { recall }}{\beta^{2} \times \text { recall }+ \text { precision }}
$$

As $\beta$ decreases, the importance of precision diminishes. To adapt this behavior to a costbased framework the $\beta$ value must depend on the cost ratio. Therefore, $\beta$ will be set as:

$$
\beta=\frac{C(+\mid-)}{C(-\mid+)}
$$

As misclassifying minority examples becomes more costly relative to majority examples, improving recall affects the $f$-measure more heavily than precision. Therefore, the minority class accuracy becomes more important as the costs become more divergent. Like the costbased wrapper, this metric is dependent on a predefined cost matrix. In the subsequent discussion we refer to the wrapper approach using $\beta$ varied $f$-measure as wrapper- $\beta$.

\subsubsection{Area Under the ROC Curve (AUROC)}

A Receiver Operating Characteristic curve (or ROC curve) is obtained by modifying the parameters of the learning algorithm to get different true positive/false positive percentages for two class problems. For classifiers which produce class probabilities, a varying threshold on the probability of belonging to a class can be applied to get the different "operating" points (e.g. a threshold of 0.3 would mean all examples classified with probability greater than 0.3 or class A are considered to have label A). After obtaining a set of points, they can be graphed. The points can be connected by lines to approximate a curve. The area under this curve provides a method for comparing classifiers (AUROC). An AUROC of one indicates a classifier which gets all of the true positives correct and has no false positives. An AUROC of 0.5 would indicate that for every true positive a classifier generates a false positive. The greater the AUROC, the better the classifier is at obtaining true positives with fewer false positives. Like the f-measure wrapper, this metric is cost independent and a classifier constructed in these terms should be flexible through a wide range of costs and distributions. In the subsequent discussion, we will refer to the wrapper approach using AUROC as wrapper-AUROC.

\subsection{A Scalable Implementation}

The Achilles heel of any wrapper system is the computation time due to the exploration of different parameters. However, the proposed wrapper framework for undersampling and 
SMOTE lends itself very well to a distributed computing paradigm. This also offers an optimal utilization of idle machines in a department or institution. We used the Condor framework (Thain et al. (2005)) to enable distributed computation on the University of Notre Dame machines resulting in significantly reduced time to obtain a final classifier ${ }^{1}$. Figure 4 shows the general implementation framework and is discussed below.

1. First, partition the data into 10-folds. Distribute each of the folds to a different computer.

2. Further partition each training fold into 5 -folds for the wrapper. Subsequently, each of the 5 -folds can also be distributed to 5 different computers.

3. The sampling parameters were used in each of the 5-folds simultaneously, and an average performance estimate was computed. First, the search for undersampling parameters was undertaken. Once the "best" undersampling parameters were found the SMOTE phase was started. Again, the SMOTE parameters were applied to each of the five folds simultaneously. The performance estimates were aggregated from each of the 5-folds, and once the best combination of undersampling and SMOTE parameters were found, the search was stopped.

We exploited this inherent parallelism in the parameter search, and were able to achieve on the order of (average) 30 times speedups as discussed in Section 5.1. It is worth noting that our largest data set contains just over 11,000 examples. Using larger data sets may affect the parallel speedup, either negatively or positively.

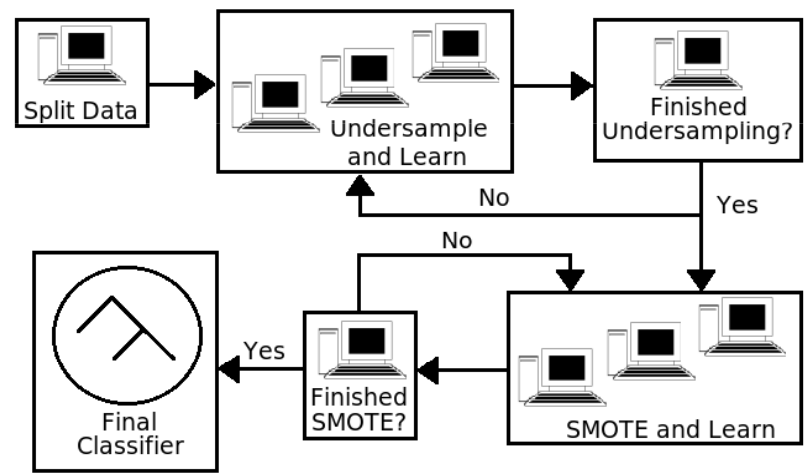

Figure 4: Distributed Wrapper Implementation.

\subsection{Classifiers}

We used two different machine learning algorithms for our experiments as base classifiers — a decision tree learner, C4.5 release 8 (Quinlan (1993)) and a rule learner, Ripper (Cohen (1995a)). We also converted the leaf frequencies produced by $\mathrm{C} 4.5$ decision trees to probability estimates after applying Laplace smoothing (Provost and Domingos (2003)).

1. Condor can be downloaded from the University of Wisconsin Condor website. 
For Ripper, the probabilities were generated based on rule confidence: the proportion of a single class against total membership. As with $\mathrm{C} 4.5$, this was modified with Laplace smoothing by introduction of a $1 / C$ class prior probability.

We also considered class prediction by setting a probability threshold. Elkan (2001) describes a system for generating optimal predictions in a two class problem based on a known cost matrix. Here, we are interested in the probability threshold for predicting one class over another. Therefore, we consider the conditions under which the expected costs for such a decision are equivalent:

$$
P(y=-\mid x) C(+\mid-)+P(y=+\mid x) C(+\mid+)=P(y=+\mid x) C(-\mid+)+P(y=-\mid x) C(-\mid-)
$$

Given that $p^{*}=P(y=+\mid x)$ and that $P(y=+\mid x)=1-P(y=-\mid x)$ under the two class assumption:

$$
\left(1-p^{*}\right) C(+\mid-)+p^{*} C(+\mid+)=p^{*} C(-\mid+)+\left(1-p^{*}\right) C(-\mid-)
$$

Rearranging this equation yields:

$$
p^{*}=\frac{C(+\mid-)-C(-\mid-)}{C(+\mid-)-C(+\mid+)+C(-\mid+)-C(-\mid-)}
$$

Under our assumptions, $C(+\mid+)=C(-\mid-)=0$ and we may therefore drop these terms from consideration.

$$
p^{*}=\frac{C(+\mid-)}{C(+\mid-)+C(-\mid+)}
$$

Therefore, when we observe a probability estimate $p$ on a testing example, an optimal decision is generated by comparing $p$ to the threshold $p^{*}$. When $p>p^{*}$, the example is labeled as the positive class, otherwise it is predicted as belonging to the negative class.

\section{Experimental Framework}

We used a variety of datasets for our study, including the KDD-99 cup data set. Eight datasets have no associated costs and we call them unfixed cost datasets. These are summarized in Table 1. Four of the datasets are from the UCI repository, and the other four are real-world examples that we acquired from different sources. To establish a context, we will briefly introduce the eight unfixed cost datasets. The fixed cost KDD-99 cup data set will be discussed separately in Section 5 .

1. The Phoneme data set is used to distinguish between nasal and oral sounds based on 5 features. The distribution is 3818 nasal and 1586 oral samples. Information in this data set comes from the ELENA project.

2. The Segment data set (D.J. Newman and Merz (1998)) originally consisted of six equally distributed classes from an image segmentation problem. We transform it into a two class problem by considering one class of size 330 examples as the minority class, and the rest of classes as a single majority class with 1980 examples. 


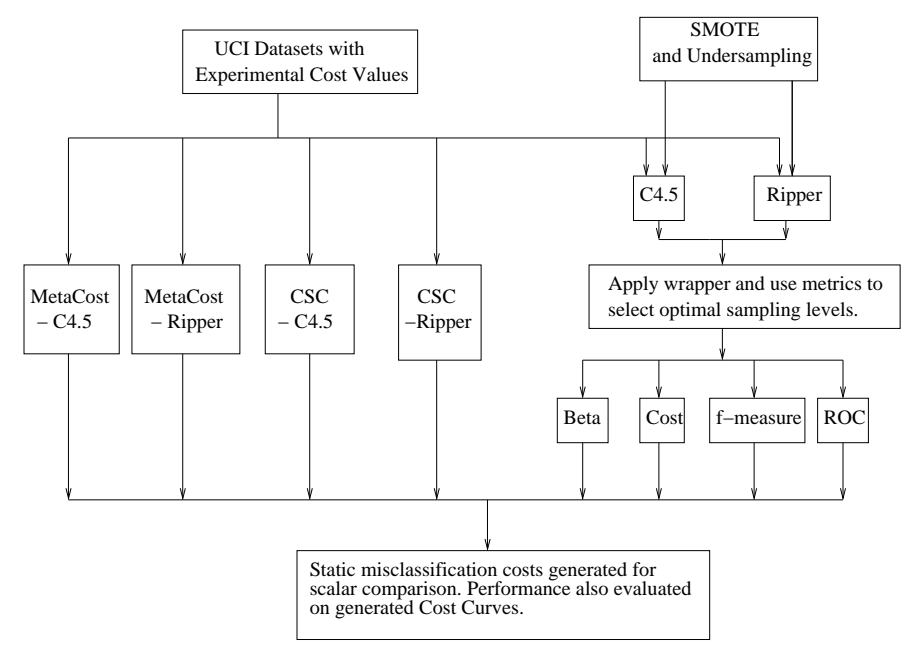

Figure 5: Experiments Overview.

3. The E-state data set (Chawla et al. (2002)) consists of electro-topological state descriptors for a series of compounds from the National Cancer Institute's Yeast AntiCancer drug screen. The activity classes are either active - at least one single yeast strain was inhibited more than $70 \%$, or inactive - no yeast strain was inhibited more than $70 \%$. The data set has 5,322 samples with 635 samples of active compounds.

4. The Page data set (Esposito et al. (1994)) consists of classifying all the blocks of a page layout of a document that has been detected by a segmentation process. This data set originally had five classes, and we have converted it to two classes: 4913 text examples and 560 non-text examples.

5. The Satimage data set (D.J. Newman and Merz (1998)) originally contained 6 classes. For our purposes, we used the two-class conversion outlined in (Provost et al. (1998)), where the smallest class receives one label and the others are part of the larger class. This produced a data set with 5809 majority class examples and 626 minority class examples.

6. Compustat North America is a database of U.S. and Canadian fundamental and market information on various active and inactive publicly held companies. It provides more than 300 annual Income Statement, Balance Sheet, Statement of Cash Flows, and supplemental data items. We extracted various financial ratios from the database, and after filtering companies based on the rating we had a total of 10,358 in the training set and 3,258 in the testing set. To make the problem resemble a real-world scenario, the training set comprised data from 1995-2001 and the testing set comprised data from 2002-2004. The interested reader can acquire the Compustat data set from the authors.

7. The Oil data set was provided by Kubat et al. (1998b). This data set has 41 oil slick samples and 896 non-oil slick samples. 


\begin{tabular}{|c|c|c|c|}
\hline Data Set & Examples & Features & Class Balance \\
\hline Phoneme & 5,404 & 5 & $79: 21$ \\
\hline Segment & 2,310 & 19 & $86: 14$ \\
\hline E-state & 5,322 & 12 & $88: 12$ \\
\hline Page & 5,473 & 10 & $90: 10$ \\
\hline Satimage & 6,435 & 36 & $90: 10$ \\
\hline Compustat & Training $=10,358 ;$ Testing $=3,258$ & 20 & $96: 4$ \\
\hline Oil & 937 & 49 & $96: 4$ \\
\hline Mammography & 11,183 & 6 & $98: 2$ \\
\hline
\end{tabular}

Table 1: Data Set distributions, in an increasing order of class imbalance.

8. The Mammography data set (Woods et al. (1993)) has 11,183 total examples with 260 calcifications. Using a trivial classifier would yield $97.68 \%$ default accuracy. However, such a classifier would most likely yield a poor total cost, particularly when the cost ratio is very skewed.

We used a 10-fold cross-validation framework for all but the Compustat data set since it was associated with a testing set collected at a different time. The results were averaged over the 5 different 10 -fold runs.

While these datasets are devoid of any known testing costs, we injected a range of cost ratios to carefully establish the generality of the wrapper framework and compare to cost-sensitive learning methods. As a convention, we will use the notation of $(F N: F P)$ to indicate the cost ratio for making a false negative error to a false positive error. For instance a ratio of $(2: 1)$ indicates that it is twice as costly to make a false negative than to make a false positive. This aligns with the premise that it is more costly to call a positive (minority) class negative than to call a negative (majority) class positive.

We will compare the wrapper and cost-based learners under several cost scenarios. First, we will consider a case where costs are known at training and testing times. Then, we will investigate a situation where costs will remain unknown at both training and testing times. Finally, we will study how well classifiers generalize throughout cost space. We will compare the impact of different evaluation criteria in the wrapper mode. We also compare both C4.5 and Ripper generated using the wrapper method with MetaCost (Domingos (1999)) and Cost-Sensitive Classifier (CSC) (Zadrozny et al. (2003a)) using the same base C4.5 and Ripper classifiers. We used the Weka implementations of MetaCost and Cost-Sensitive Classifier (Witten and Frank (2005)). Figure 5 provides an overview of our experiments.

\section{Results: Unfixed Cost Datasets}

We will first discuss the analysis using AUROC and f-measure and then present the costbased results. 
Table 2: Average sampling levels and 10-fold performance measures for a (1:1) cost matrix (equal costs of errors) with the C4.5 classifier, including the standard deviations. The results are averaged over the five different random runs. Wrapper Evaluation indicates the evaluation function used for guiding the wrapper mode. The SMOTE and Undersample columns indicate the amounts of SMOTE and undersampling discovered by the wrapper. The subsequent columns indicate the evaluation function used on the testing set. Base indicates the base classifier without any sampling. The best performance is shown in bold.

\begin{tabular}{|c|c|c|c|c|c|c|}
\hline & Wrapper- & S & US & Cost & f-measure & AUROC \\
& Evaluation & & & & & \\
\hline \multirow{3}{*}{ Phoneme } & Base & - & - & $364 \pm 50$ & $0.772 \pm 0.032$ & $0.905 \pm 0.018$ \\
& f-meas & 212 & 6 & $415 \pm 53$ & $\mathbf{0 . 7 7 8} \pm \mathbf{0 . 0 2 5}$ & $\mathbf{0 . 9 2 2} \pm \mathbf{0 . 0 1 7}$ \\
& AUROC & 562 & 2 & $436 \pm 51$ & $0.772 \pm 0.024$ & $0.921 \pm 0.019$ \\
\hline \hline \multirow{3}{*}{ Segment } & Base & - & - & $9 \pm 6$ & $0.973 \pm 0.018$ & $0.957 \pm 0.011$ \\
& f-meas & 460 & 8 & $5 \pm 3$ & $\mathbf{0 . 9 8 5} \pm \mathbf{0 . 0 1 2}$ & $\mathbf{0 . 9 6 6} \pm \mathbf{0 . 0 0 8}$ \\
& AUROC & 420 & 13 & $7 \pm 4$ & $0.977 \pm 0.012$ & $\mathbf{0 . 9 6 6} \pm \mathbf{0 . 0 0 8}$ \\
\hline \hline \multirow{3}{*}{ E-State } & Base & - & - & $319 \pm 5$ & $0.003 \pm 0.009$ & $0.495 \pm 0.006$ \\
& f-meas & 640 & 20 & $1176 \pm 297$ & $0.227 \pm 0.018$ & $0.580 \pm 0.030$ \\
& AUROC & 1030 & 21 & $1298 \pm 276$ & $\mathbf{0 . 2 4 2} \pm \mathbf{0 . 0 2 1}$ & $\mathbf{0 . 5 9 7} \pm \mathbf{0 . 0 2 7}$ \\
\hline \hline \multirow{3}{*}{ Page } & Base & - & - & $78 \pm 19$ & $\mathbf{0 . 8 5 7} \pm \mathbf{0 . 0 3 9}$ & $0.959 \pm 0.013$ \\
& f-meas & 200 & 3 & $86 \pm 22$ & $0.856 \pm 0.033$ & $\mathbf{0 . 9 7 2} \pm \mathbf{0 . 0 0 4}$ \\
& AUROC & 690 & 3 & $94 \pm 22$ & $0.847 \pm 0.033$ & $\mathbf{0 . 9 7 2} \pm \mathbf{0 . 0 0 6}$ \\
\hline \hline \multirow{3}{*}{ Satimage } & Base & - & - & $259 \pm 19$ & $0.569 \pm 0.029$ & $0.900 \pm 0.019$ \\
& f-meas & 622 & 6 & $275 \pm 27$ & $0.609 \pm 0.036$ & $0.919 \pm 0.010$ \\
& AUROC & 644 & 4 & $258 \pm 16$ & $\mathbf{0 . 6 2 8} \pm \mathbf{0 . 0 2 6}$ & $\mathbf{0 . 9 2 6} \pm \mathbf{0 . 0 1 4}$ \\
\hline \hline \multirow{3}{*}{ Oil } & Base & - & - & $25 \pm 6$ & $0.314 \pm 0.256$ & $0.631 \pm 0.119$ \\
& f-meas & 260 & 2 & $21 \pm 7$ & $0.467 \pm 0.119$ & $0.722 \pm 0.110$ \\
& AUROC & 300 & 15 & $22 \pm 8$ & $\mathbf{0 . 5 1 8} \pm \mathbf{0 . 1 3 3}$ & $\mathbf{0 . 7 3 6} \pm \mathbf{0 . 1 0 1}$ \\
\hline \hline \multirow{3}{*}{ Mammo. } & Base & - & - & $76 \pm 15$ & $0.644 \pm 0.101$ & $0.863 \pm 0.031$ \\
& f-meas & 200 & 2 & $92 \pm 19$ & $\mathbf{0 . 6 7 2} \pm \mathbf{0 . 0 6 3}$ & $0.896 \pm 0.035$ \\
& AUROC & 720 & 14 & $120 \pm 29$ & $0.619 \pm 0.058$ & $\mathbf{0 . 9 0 7} \pm \mathbf{0 . 0 3 8}$ \\
\hline \hline
\end{tabular}


Table 3: Average sampling levels and 10-fold performance measures for a $(1: 1)$ cost matrix (equal costs of errors) with the Ripper classifier, including the standard deviations. The results are averaged over the five different random runs. Wrapper Evaluation indicates the evaluation function used for guiding the wrapper mode. The SMOTE and Undersample columns indicate the amounts of SMOTE and undersampling discovered by the wrapper. The subsequent columns indicate the evaluation function used on the testing set. Base indicates the base classifier without any sampling. The best performance is shown in bold.

\begin{tabular}{|c|c|c|c|c|c|c|}
\hline & $\begin{array}{c}\text { Wrapper- } \\
\text { Evaluation }\end{array}$ & SM & US & Cost & f-measure & AUROC \\
\hline \hline \multirow{3}{*}{ Phoneme } & Base & - & - & $385 \pm 45$ & $0.748 \pm 0.032$ & $0.823 \pm 0.022$ \\
& f-meas & 150 & 9 & $491 \pm 39$ & $0.753 \pm 0.017$ & $0.899 \pm 0.015$ \\
& AUROC & 162 & 6 & $481 \pm 59$ & $\mathbf{0 . 7 5 9} \pm \mathbf{0 . 0 2 5}$ & $\mathbf{0 . 9 0 2} \pm \mathbf{0 . 0 2 0}$ \\
\hline \hline \multirow{3}{*}{ Segment } & Base & - & - & $9 \pm 4$ & $0.971 \pm 0.014$ & $0.956 \pm 0.007$ \\
& f-meas & 300 & 5 & $6 \pm 4$ & $\mathbf{0 . 9 8 2} \pm \mathbf{0 . 0 1 5}$ & $\mathbf{0 . 9 6 8} \pm \mathbf{0 . 0 0 6}$ \\
& AUROC & 460 & 9 & $7 \pm 5$ & $0.979 \pm 0.016$ & $0.967 \pm 0.007$ \\
\hline \hline \multirow{3}{*}{ E-State } & Base & - & - & $318 \pm 4$ & $0.000 \pm 0.000$ & $0.493 \pm 0.000$ \\
& f-meas & 520 & 24 & $1501 \pm 372$ & $\mathbf{0 . 2 3 6} \pm \mathbf{0 . 0 1 8}$ & $\mathbf{0 . 5 7 9} \pm \mathbf{0 . 0 2 9}$ \\
& AUROC & 660 & 18 & $1480 \pm 160$ & $0.235 \pm 0.012$ & $0.574 \pm 0.018$ \\
\hline \hline \multirow{3}{*}{ Satimage } & Base & - & - & $78 \pm 16$ & $0.859 \pm 0.026$ & $0.906 \pm 0.017$ \\
& f-meas & 190 & 5 & $84 \pm 16$ & $\mathbf{0 . 8 6 2} \pm \mathbf{0 . 0 2 4}$ & $0.966 \pm 0.011$ \\
& AUROC & 680 & 14 & $99 \pm 25$ & $0.845 \pm 0.036$ & $\mathbf{0 . 9 6 9} \pm \mathbf{0 . 0 0 6}$ \\
\hline \hline \multirow{3}{*}{ Oil } & Base & - & - & $226 \pm 23$ & $0.571 \pm 0.055$ & $0.720 \pm 0.029$ \\
& f-meas & 400 & 6 & $263 \pm 47$ & $\mathbf{0 . 6 4 5} \pm \mathbf{0 . 0 4 7}$ & $0.918 \pm 0.017$ \\
& AUROC & 488 & 7 & $287 \pm 48$ & $0.633 \pm 0.037$ & $\mathbf{0 . 9 2 5} \pm \mathbf{0 . 0 1 3}$ \\
\hline \hline \multirow{3}{*}{ Mammo. } & Base & - & - & $24 \pm 8$ & $0.270 \pm 0.249$ & $0.529 \pm 0.122$ \\
& f-meas & 310 & 6 & $21 \pm 9$ & $0.521 \pm 0.180$ & $0.695 \pm 0.092$ \\
& AUROC & 570 & 11 & $21 \pm 10$ & $\mathbf{0 . 5 7 2} \pm \mathbf{0 . 1 5 5}$ & $\mathbf{0 . 7 2 3} \pm \mathbf{0 . 1 1 0}$ \\
\hline \hline & Base & - & - & $78 \pm 16$ & $0.648 \pm 0.096$ & $0.756 \pm 0.052$ \\
& AUROC & 180 & 5 & $81 \pm 14$ & $\mathbf{0 . 6 9 9} \pm \mathbf{0 . 0 4 7}$ & $0.869 \pm 0.023$ \\
& 240 & 9 & $92 \pm 22$ & $0.675 \pm 0.061$ & $\mathbf{0 . 8 7 4} \pm \mathbf{0 . 0 2 6}$ \\
\hline
\end{tabular}




\subsection{AUROC and F-measure analysis}

Our first experiment considered the effect of using $A U R O C$ and $f$-measure for guiding the wrapper search under a $(1: 1)$ cost environment. This experiment allowed us to observe the interaction between an objective function for the wrapper, sampling level, and an evaluation metric on the testing set. Tables 2 and 3 summarize the results with C4.5 and Ripper as base classifiers, respectively. The results are averaged over the 50 runs ( 5 different random trials and 10-fold CV during each trial). The standard deviations are also shown beside the averaged performance measures. The cost column represents the cost of making errors at $(1: 1)$; thus, it is essentially the total number of errors. For instance, for the Mammography data set if $A U R O C$ is used with the wrapper, the amount of SMOTE is $720 \%, 14 \%$ of the majority class is removed, and the testing set f-measure is 0.619 and $A U R O C$ is 0.907 .

We found that SMOTE and undersampling generally help the base classifiers learned on the different datasets. The results carry more significance for Ripper. Irrespective of the wrapper evaluation function, the sampling methods always result in an improved AUROC over the base classifier. In all but two cases, the $f$-measure is also improved. The two deviations from the norm are the Page and Mammography data sets. While Page had marginal deterioration over the base classifier, the wrapper-AUROC on the Mammography data set results in a $2.5 \%$ drop over the base classifier for the $f$-measure. However, AUROC is always improved. We believe this to be an artifact of a higher FPrate as we focus on the minority class being less detrimental.

We also find that there is not a distinct positive correlation between the $f$-measure in the wrapper-mode and corresponding improvements in the final evaluation. For instance, C4.5 improved the $f$-measure on four datasets when using AUROC as the wrapper evaluation metric rather than the $f$-measure. The question then becomes, why does this happen with the $f$-measure and not with $A U R O C$ ? We attribute this to the nature of the two metrics. The $f$-measure is tuned to a fixed threshold of 0.5. This means that the fixed point threshold of 0.5 can become unstable. We believe this results in more generalized performances when using $A U R O C$ as the wrapper evaluation function. Essentially, our goal was to identify which one was less prone to reaching a local maximum in the wrapper scheme.

Both wrapper-AUROC and wrapper-fmeasure generally result in different amounts of SMOTE and undersampling, as they optimize different properties of the classifier. As expected using different classifiers also results in different levels of sampling. It also seems that the amount of SMOTE or undersampling is not dependent on the class skew, but on the properties of the feature space. Consider the E-state data set, which has the largest amount of SMOTE and undersampling and yet it is certainly not the most unbalanced data set. If we look at the statistics, E-state for both $\mathrm{C} 4.5$ and Ripper has a very poor $f$-measure, and an AUROC indicative of the random performance. However, there is a significant improvement offered by the sampling methods. Hence, we believe that a wrapper method can allow one to empirically discover the relevant amounts of sampling, as it is certainly intrinsically tied in with the data properties. Lastly, as expected using the base classifier for the $(1: 1)$ cost ratio is often beneficial in terms of improving cost, since both FP and FN are equally weighted. The error driven base classifier reduces the total number of FP and FN. 
Table 4: Time in seconds by different approaches. SEQ: Sequential version; DIST: Distributed version.

\begin{tabular}{|c|l|l||l|l|}
\hline Dataset & $\begin{array}{l}\text { wrapper- } \\
\text { AUROC SEQ } \\
(\mathrm{C} 4.5)\end{array}$ & $\begin{array}{l}\text { wrapper- } \\
\text { AUROC DIST } \\
(\mathrm{C} 4.5)\end{array}$ & $\begin{array}{l}\text { wrapper- } \\
\text { AUROC SEQ } \\
\text { (Ripper) }\end{array}$ & $\begin{array}{l}\text { wrapper- } \\
\text { AUROC DIST } \\
\text { (Ripper) }\end{array}$ \\
\hline Compustat & 1,061 & 22 & 3,353 & 89 \\
\hline Estate & 3,028 & 71 & 727 & 22 \\
\hline Mammography & 181 & 7 & 308 & 14 \\
\hline Oil & 30 & 2 & 45 & 2 \\
\hline Page & 75 & 9 & 276 & 16 \\
\hline Phoneme & 1,816 & 87 & 673 & 33 \\
\hline Satimage & 2,797 & 105 & 3,010 & 81 \\
\hline Segment & 726 & 33 & 120 & 5 \\
\hline \hline Average & $1,214.25$ & 42 & 1,064 & 32 \\
\hline
\end{tabular}

\subsection{Timing}

Table 4 shows the average time (in seconds) taken on a dataset for a single fold. We show the time taken by both the sequential and distributed versions of the wrapper framework. As a representative of all the wrappers, we show the timings of the wrapper-AUROC method, especially given that this results in larger amounts of sampling than the wrapper-fmeasure. Exactly the same underlying code-base is part of the sequential and distributed runs. The distributed implementations provide a significant improvement in timing, making the wrapper a very competitive approach. On an average, it takes only 42 seconds with C4.5 and 32 seconds with Ripper to complete the wrapper search, which is very encouraging especially in the light of performance improvements.

We note that the wrapper with Ripper is faster than the wrapper with C4.5 because of the relatively lower amounts of undersampling and SMOTE with Ripper as the base classifier. The time taken for the entire wrapper paradigm is largely a function of the size of minority class examples and the associated learning complexity, which drives the search for the SMOTE amount.

\subsection{Comparison to Cost-sensitive Classifiers}

We wanted to examine an empirical relationship between learning from imbalanced datasets and cost-sensitive classification. Can we effectively handle the problem of imbalance and achieve lower costs during testing than cost-sensitive classifiers?

We compare the wrapper-induced classifiers with MetaCost (Domingos (1999)) and CostSensitiveClassifier (CSC) (Zadrozny et al. (2003b)). MetaCost wraps a "meta-learning" stage around an error-based classifier to effectively minimize cost. It estimates the conditional probability distributions of a data set via bagging (Breiman (1996)) Combining probability estimates with a simple expected-cost framework, MetaCost selects the class as $\operatorname{argmin}_{i} \sum_{j} P(y=j \mid x) C(i \mid j)$. This essentially relabels a class in the training data such 
that the expected cost is minimized. A classifier is then learned on the relabeled data and evaluated on the testing set.

The CSC method draws a new training set in accordance with each example's misclassification cost. However, when costs are highly skewed, overfitting tends to occur as a limited number of examples are resampled heavily. To counteract this, rejection sampling is used. The newly drawn training set keeps a selected example with a probability of $\frac{c}{Z}$ where $c$ is the examples misclassification cost and $Z$ is a selected constant greater than the maximum cost of the original training set.

We show comparisons using two illustrations. First, we use cost trends where the $y$-axis indicates the total cost as the cost ratio varies on the $x$-axis. Second, we use the cost curves Drummond and Holte (2006).

\subsubsection{Cost Trends}

Here, we compare the wrapper framework for countering class imbalance with the MetaCost and Cost-Sensitive Classifier (CSC). These classifiers require specific tuning to a cost ratio $(F N: F P)$, which we have varied as $(2: 1),(5: 1),(10: 1)$, and $(20: 1)$. We use these cost ratios, as these have been previously considered in other studies (Domingos (1999); Drummond and Holte (2006)).

If the classifiers were built without utilization of a cost-matrix, then the generated posterior probabilities were thresholded at the corresponding cost ratios for the testing set. This scenario can be used when the costs are completely unknown during training. The classifiers generate a posterior probability distribution on the testing set. Depending on the cost environment during the testing stage, the probabilities can effectively be thresholded to reflect the cost distributions. However, the classifiers are not re-learned. The base classifier, wrapper-fmeasure, and wrapper-AUROC fall under this umbrella. This allows us to directly compare the effectiveness of dealing with imbalance without incorporating costs during the training phase. We would like to point the reader to Appendix A, which shows the benefits from thresholding to reflect the costs after applying SMOTE and undersampling. On the other hand, if the classifiers incorporated cost during learning, such as wrappercost, wrapper- $\beta$, MetaCost and CSC, then no thresholding was performed. This is under the assumption that the classifiers have already been optimized on an objective function attuned to a particular cost. Thus, we generated multiple such classifiers for the different cost ratios.

Figures 6 and 7 shows the range of costs obtained across the eight datasets, for different cost ratios. The $\mathrm{x}$-axis in the Figure shows the different datasets and the $\mathrm{y}$-axis shows the normalized costs. We normalized the costs between 0 and 1 to enable a better display of results.

The results show an interesting trend. At $(2: 1)$, the cost-sensitive classifiers generally resulted in lower costs than the wrapper based methods. However, as we increased the cost ratios, particularly at $(10: 1)$ and $(20: 1)$, the wrapper based methods begin to result in lower costs than the cost-sensitive classifiers that are even trained on the corresponding costs. The wrapper-AUROC and wrapper-fmeasure make a very compelling case at higher costs; without using costs during training, they are able to often outperform the costsensitive classifiers. The results hold with both $\mathrm{C} 4.5$ and Ripper. Thus, a combination 


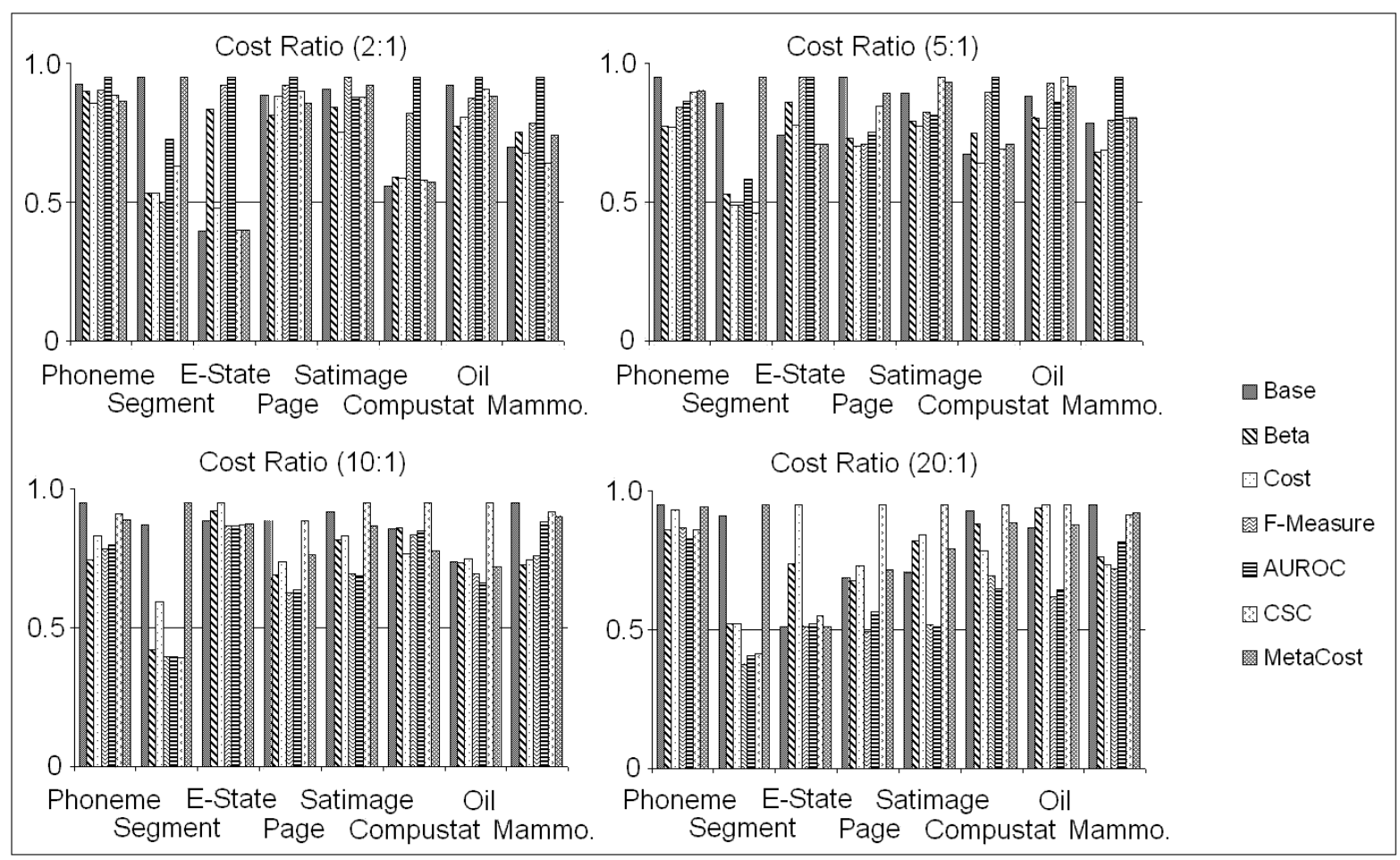

Figure 6: Cost trends with $\mathrm{C} 4.5$ as base classifier. Beta is wrapper- $\beta$ and Cost is wrappercost.

of the sampling approaches for countering class imbalance and thresholding is effective in overcoming the cost distributions. In fact we found that the wrapper-AUROC and wrapperfmeasure are quite effective across the cost ranges. We conjecture this to be an outcome of an improvement in the quality of estimates, thus resulting in the reduced overall costs (Cieslak and Chawla (2006)). The effective treatment of imbalance without using costs during training can result in robust posterior probability estimates applicable to a variety of cost scenarios during testing. This is certainly desirable, especially when the costs are unknown or can change during testing. Between C4.5 and Ripper, the former is more effective as a classifier in reducing the costs during testing. Lastly, all the methods result in lower costs over the base classifier at cost ratios $>(2,1)$. Thus, treating for imbalance or changing the objective function to be cost-sensitive is always preferred over the thresholded estimates from a base classifier.

Zhou and Liu (2006) found that there might be a disconnect between learning from imbalanced datasets and cost-sensitive learning, which is contrary to our observations. However, we believe the differences in their conclusions can be attributed to a lack of sufficient search in the sampling parameter space. They resort to "default" sampling parameters, but, as we point out, there are really no "default" sampling parameters for a data set and a classifier. We show that using wrapper-induced sampling methods on a multi-class data set, we were able to achieve lower costs than any other known method. Lastly, their cost 


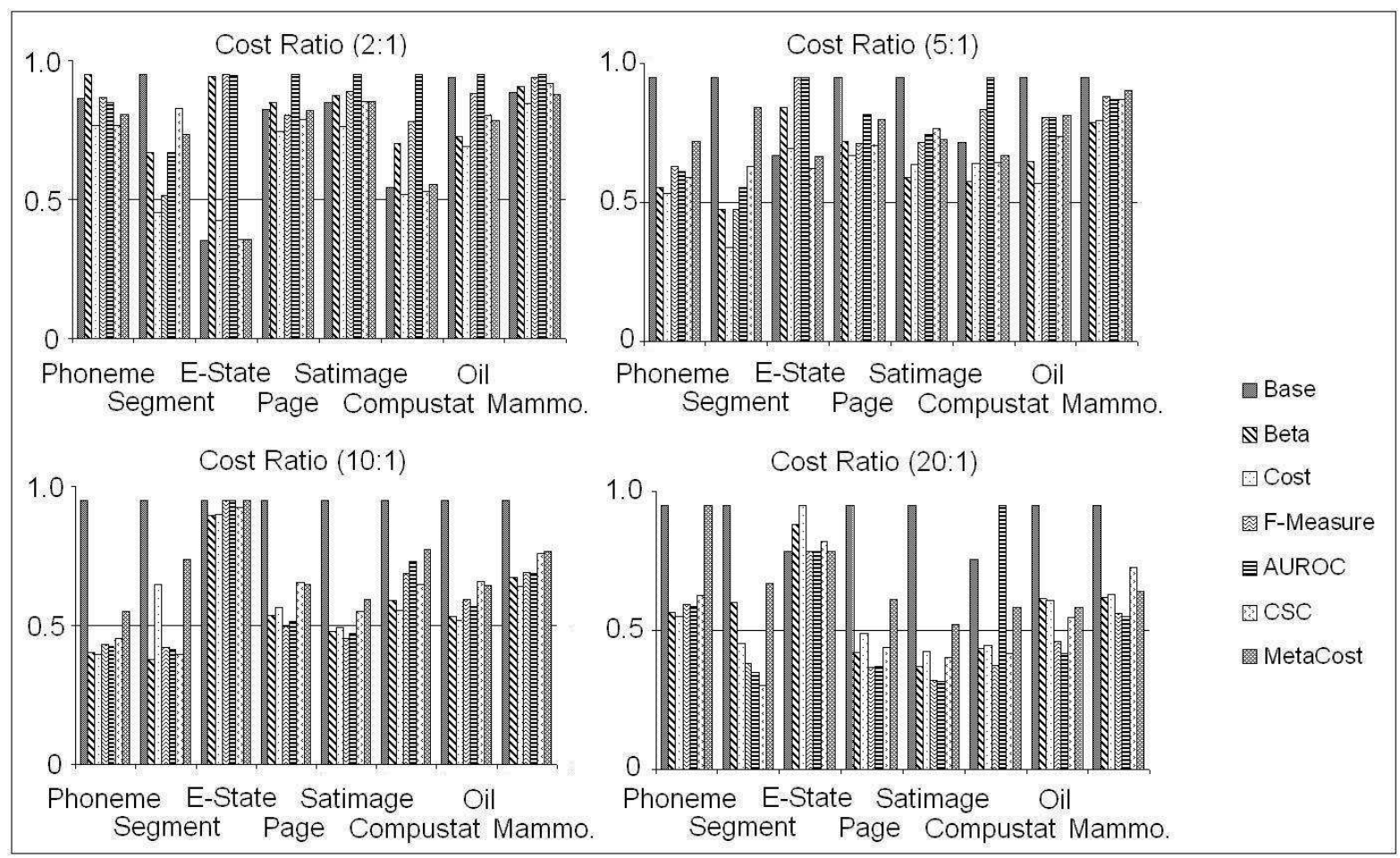

Figure 7: Cost trends with Ripper as base classifier. Beta is wrapper- $\beta$ and Cost is wrappercost.

functions were constrained to 1:10, while we extended our study to 1:100. However, our comprehensive experiments on real-world datasets, including the to be discussed, multiclass KDD-99 Cup dataset, demonstrate that treating a data set for imbalance first is often beneficial compared with cost-sensitive classifiers.

\subsubsection{Cost Curves}

Conditioning on a particular T Prate or FPrate cut-off may not immediately establish the expected generalization capacity of a classifier. It may be more appropriate to gauge the performance of classifiers across a range of TPrate and FPrate. Thus, cost curves (Drummond and Holte (2006)) founded on expected cost may yield a clearer understanding of the classifiers within that operating environment. These curves plot performance (expected cost normalized between 0 and 1 ) on the $\mathrm{Y}$-axis. This function is given by:

$$
N E[C]=(1-\text { TPrate }- \text { FPrate }) \times P C F(+)+F P
$$

The $\mathrm{X}$-axis combines cost and class distribution in the following manner:

$$
P C F(+)=\frac{p(+) C(-\mid+)}{p(+) C(-\mid+)+p(-) C(+\mid-)}
$$


a) Phoneme

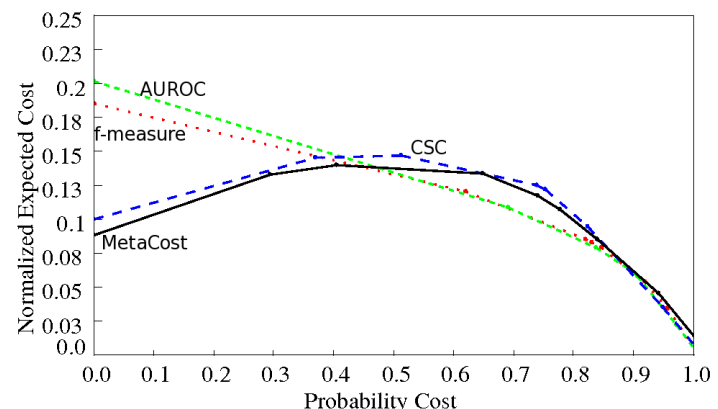

c) E-State

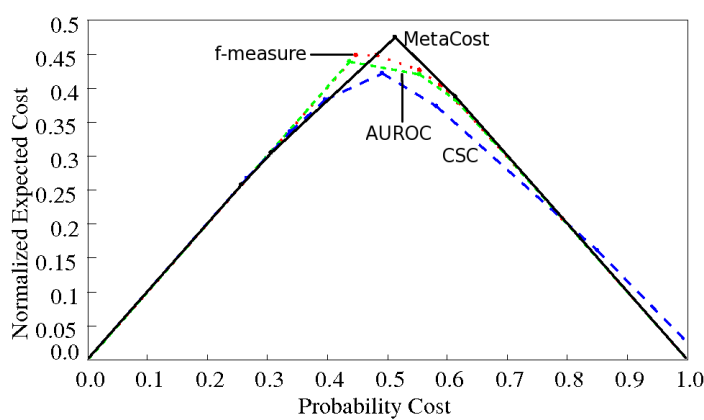

e) Satimage

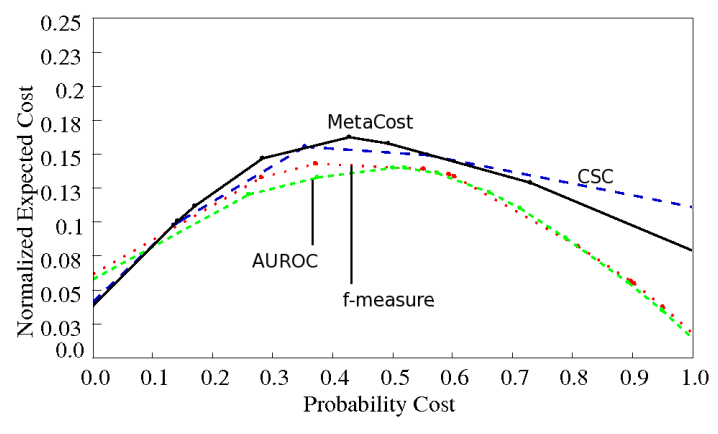

g) Oil

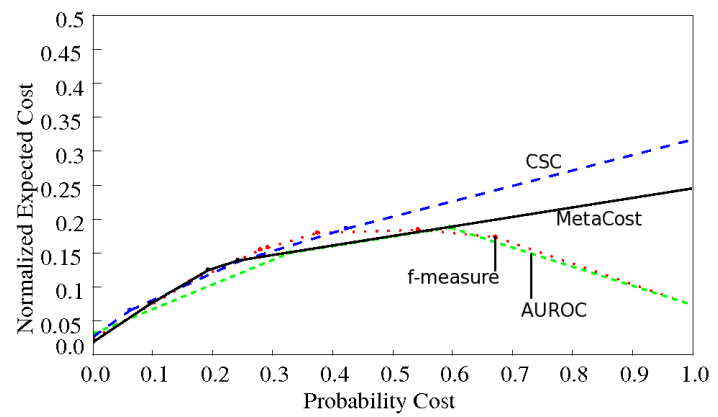

b) Segment

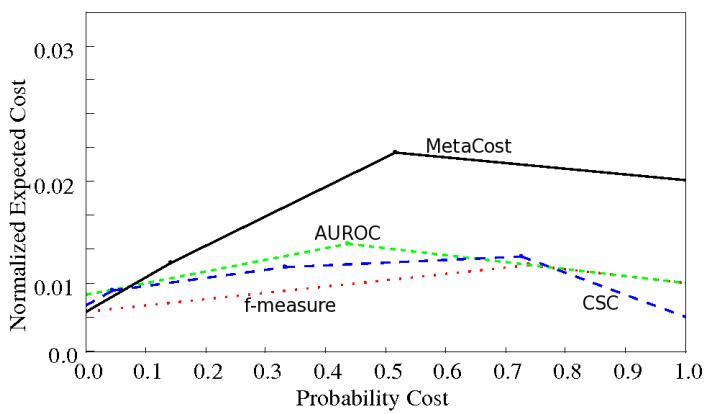

d) Page

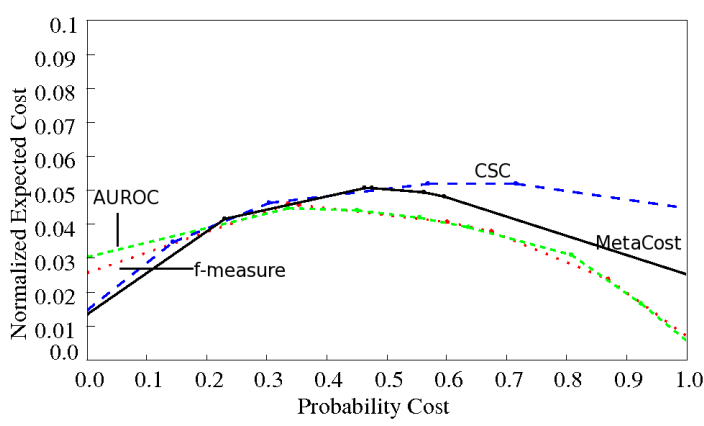

f) Compustat

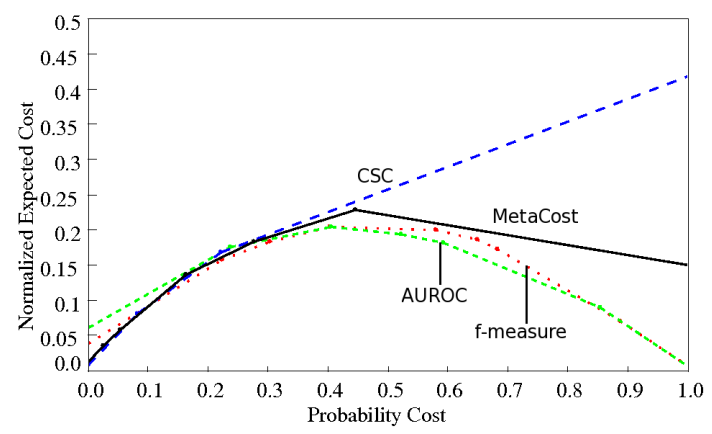

h) Mammography

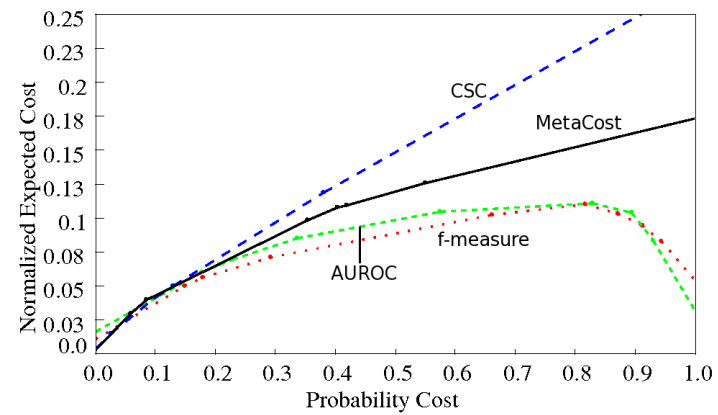

Figure 8: C4.5 Cost Curves. 
a) Phoneme

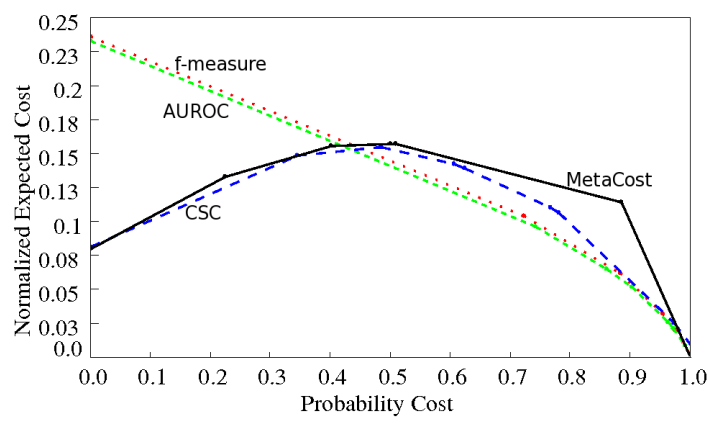

c) E-State

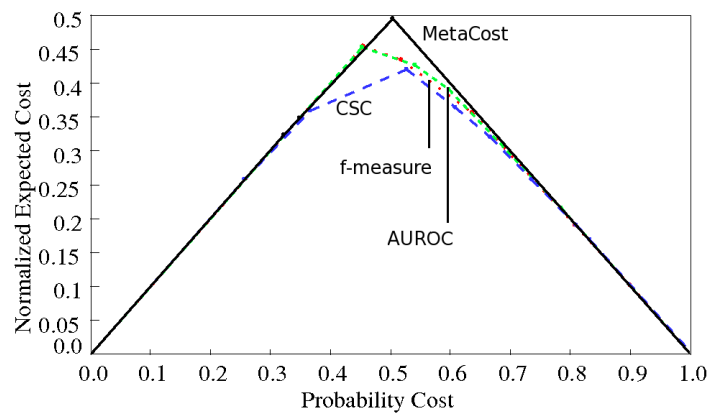

e) Satimage

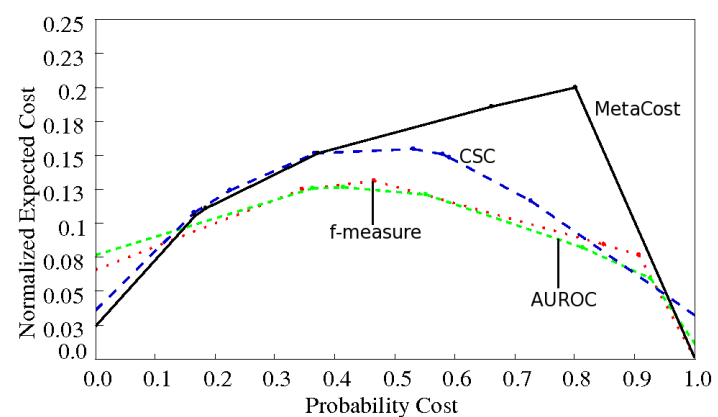

g) Oil

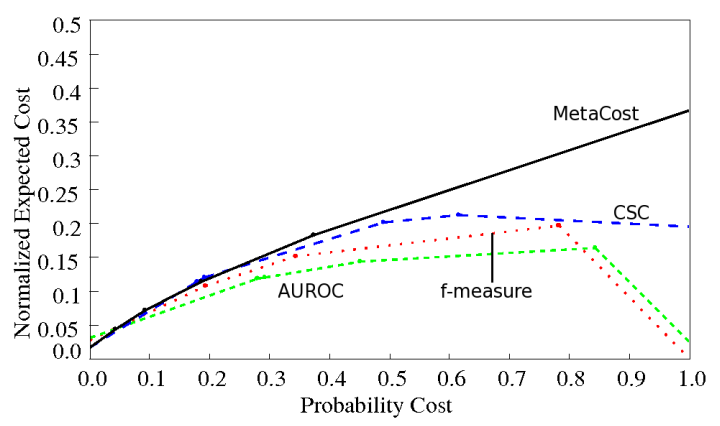

b) Segment

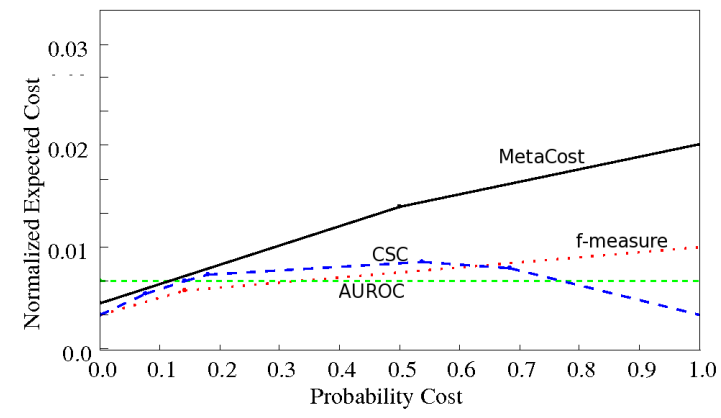

d) Page

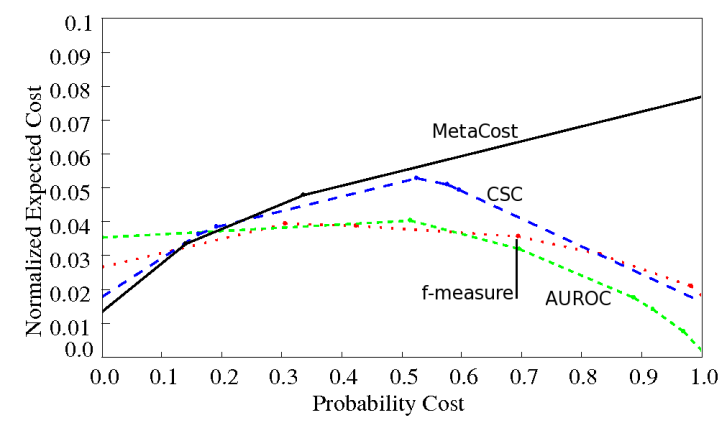

f) Compustat

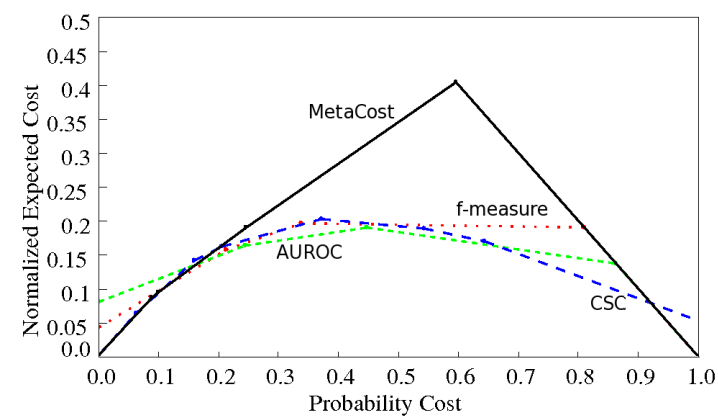

h) Mammography

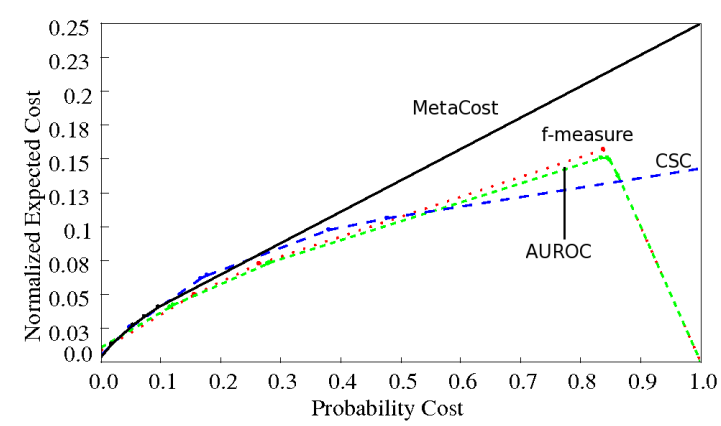

Figure 9: Ripper Cost Curves. 
where $C(-\mid+)$ is the cost of misclassifying a positive example as negative, $C(+\mid-)$ is the cost of misclassifying a negative example as positive, $p(+)$ is the probability of a positive example and $p(-)=1-p(+)$ is the probability of a negative example. This is a multi-faceted tool which enables sophisticated analysis of classifier performance under fluid conditions. Overall, cost curves allow visualization of the interplay of classifiers as the weighted importance of two classes wax and wane. There is a correspondence of a point in ROC space to a line in cost space. A single (TPrate, F Prate) pair from the ROC space gets translated to a line segment in the cost space. Thus, different operating points from the ROC curve will represent different lines in the cost space.

For clarity in presentation, we show the lower envelopes of the cost curves of the following four methods: wrapper-fmeasure, wrapper-AUROC, CSC, and MetaCost. We wanted to draw our comparisons between using classifiers optimized on cost-insensitive measures and cost-sensitive classifiers. We constructed the cost curves as follows. The cost-sensitive classifiers - MetaCost and CSC - are trained with the different cost ratios, drawn from the following $F N: F P-1: 1,2: 1,5: 1,10: 1,20: 1,30: 1,40: 1, \ldots 100: 1$. This resulted in 13 pairs of (T Prate, F Prate) for each of the above methods or a total of $91(13 \times 7)$ cost curves. After plotting all the possible cost curves from different methods, we only retained their corresponding lower envelopes to reflect their best operating ranges.

Figures 8 and 9 show the cost curves. The $\mathrm{x}$-axis in the Figures represents the Probability Cost Function (PCF) and the y-axis represents Normalized Expected Cost (NEC). As seen in the Figures, the methods start-off overlapping with each other, but as the PCF increases they begin to separate. Based on the comparisons across all the datasets, we find that treating the datasets for imbalance using the wrapper mode results in the most effective sets of classifiers across the range of $\mathrm{PCF}$. If a classifier produces the lowest envelope it is the most optimal across the entire range of PCF's. Our observations corroborate the findings in the previous subsection. The lower envelopes of the cost-insensitive measures generally dominate over the cost-sensitive classifiers, especially at higher costs. Thus, using wrappers to first counter the class imbalance problem was generally more cost-effective than the cost-sensitive classifiers. We also found that wrapper-AUROC were either comparable to or better than wrapper-fmeasure, and can be the recommended optimization criterion. Between MetaCost and CSC, we found MetaCost to result in lower costs than CSC using $\mathrm{C} 4.5$ as the base- classifier. Between wrapper-AUROC and MetaCost, the former resulted in a consistently lower envelope for Mammography, Compustat, and Satimage.

\section{Fixed Cost KDD Cup Intrusion Data Set}

In addition to cases of imbalanced class, we are also interested in studying classifier performance on datasets of known cost. Therefore, we have investigated a data set from the 1999 KDD Cup, Intrusion, which forces extension of our work from two-class to a multi-class problem. We demonstrate our results exceed those of the champion.

We consider this data set in a way that allows for comparisons with previously published work. A particularly interesting example for comparison is to look at the results from of the KDD-99 cup data. A cost matrix was used in the scoring of the competition as shown in Table 5 (Elkan (1999)). This produced the results shown in Table 6 below. There were many duplicate examples in the original 5 million example training set. All duplicate examples 
Table 5: Cost matrix used for scoring entries in KDD-99 CUP competition.

\begin{tabular}{|c|c|c|c|c|c|}
\hline Actual/predicted & dos & $\mathrm{u} 2 \mathrm{r}$ & $\mathrm{r} 2 \mathrm{l}$ & probe & normal \\
\hline dos & 0 & 2 & 2 & 1 & 2 \\
\hline $\mathrm{u} 2 \mathrm{r}$ & 2 & 0 & 2 & 2 & 3 \\
\hline $\mathrm{r} 2 \mathrm{l}$ & 2 & 2 & 0 & 2 & 4 \\
\hline probe & 2 & 2 & 2 & 0 & 1 \\
\hline normal & 2 & 2 & 2 & 1 & 0 \\
\hline
\end{tabular}

Table 6: Comparison of results obtained on the original KDD CUP 99 test data. The numbers beside $\mathrm{u} 2 \mathrm{r}$ and $\mathrm{r} 2 \mathrm{l}$ indicate the SMOTE percentage utilized for the experiments.

\begin{tabular}{|c|c|}
\hline & Cost per test example \\
\hline Winning Strategy (Elkan (1999)) & 0.2331 \\
\hline Decision Tree (Amor et al. (2004)) & 0.2371 \\
\hline Naive Bayes (Amor et al. (2004)) & 0.2485 \\
\hline Multi-classifier (Sabhnani and Serpen (2003)) & 0.2285 \\
\hline Using C4.5 on Training Data 1 u2r (200) - r2l (0) & 0.2478 \\
\hline Using RIPPER on Training Data 1 u2r (100) - r2l (0) & 0.2444 \\
\hline Using C4.5 on Training Data 2 u2r (200) - r2l (300) & 0.2051 \\
\hline Using RIPPER on Training Data 2 u2r (100) - r2l (0) & $\mathbf{0 . 2 0 4 9}$ (Chawla et al. (2005)) \\
\hline
\end{tabular}

were removed. We also undersampled both the normal and neptune (dos) class by removing examples which occurred only once. For Training Data 1 as in Table 5, we undersampled the normal class, and for the Training Data 2 we undersampled both the normal and neptune classes. Note that for both of these sets of experiments, the test set remained unchanged. Our assumption was that some of the rarely occurring training examples could be mislabeled or they were not very representative. Table 6 shows the results.

It can be seen that our approach with RIPPER as the classifier produced the lowest cost per example after undersampling both the normal and neptune classes (Training Data 2), and applying $100 \%$ SMOTE to the u2r class, while keeping the r2l class unchanged. This was better than the winner of the contest and better than the subsequent results from the literature. Also using C4.5 as the base classifier with SMOTE (200\% u2r and 300\% for r2l) resulted in lower cost than the other published techniques. We also achieve better results than the ones achieved by Zhou and Liu (2006).

\section{Conclusions}

In this work, a wrapper approach was utilized to determine the percentage of minority examples to add to the training set and the percentage for undersampling the majority class examples. We used a variety of datasets with different class distributions and characteristics, including a number of real world datasets. The wrapper approach works by performing a guided search of the parameter space. The evaluation function was applied with a five- 
fold cross-validation on the training set. Once the best percentages for undersampling and SMOTE are found they can be used to build a classifier on the updated training set and applied on the unseen testing set. We have demonstrated the ability to optimize sampling levels for an evaluation function, resulting in effective generalization performance.

We also wished to view the impact of using a threshold function on the probabilities produced by a classifier to generate optimal decisions in a cost-based problem. As we note in Appendix A, cost thresholding works particularly well at reducing the total cost of a classifier, particularly when operating at a higher cost ratio. Thus, we may assume that sampling has allowed classifiers to develop a calibration of probabilities better suited for higher cost ratios.

Our conclusions, connected to the questions posited in the Introduction, can be summarized as follows.

- The proposed wrapper-based paradigm, optimizing on different evaluation functions, was effective in automatically discovering the potentially optimal amounts of sampling for a data set. This was effective in countering the cost-imbalance during training and testing as well. We showed that the classifiers learned on sampling parameters discovered by optimizing on metrics transparent to costs, such as AUROC and $f$ measure, retain their effectiveness when costs were introduced during the testing stage. Both the metrics were very effective compared to the base classifier as demonstrated by the $A U R O C$ and $f$-measure on the testing sets. Thus, changing the data distribution is important to learning the classifiers in an imbalanced domain.

- We also compared our approaches to cost-sensitive learners. In an analysis of raw total cost, we found a strong tendency for wrapper-AUROC and wrapper-fmeasure to generally outperform the other wrapper evaluation functions. Moreover, at higher cost ratios they also dominated over CSC and MetaCost.

Another set of comparisons among the classifiers was done using cost curves. Using the lower envelope formed by multiple classifiers learned at different cost ratios, it was also possible to examine each evaluation metric and the cost-based learners throughout cost space. In this section, we have noted that wrapper-AUROC tends to produce classifiers that result in more effective generalization in the PCF space, even better than the cost-sensitive classifiers optimized at different cost ratios.

- We also showed that a combination of undersampling and SMOTE offers a significant advantage over several cost-based classifiers in a number of realistic cost environments, including a champion level performance on a KDD Cup Challenge.

\section{Acknowledgments}

We are grateful to Robert Holte for providing the Cost Curves software and Oil data set. David Cieslak was partially supported by the the Arthur J. Schmitt Fellowship. 


\begin{tabular}{|c|c|c|c|c|c|c|}
\hline & & $(1: 1)$ & $(2: 1)$ & $(5: 1)$ & $(10: 1)$ & $(20: 1)$ \\
\hline \multirow{4}{*}{ Phoneme } & AUROC thresholded & 3495 & 4395 & 6005 & 7410 & 9125 \\
\hline & AUROC unthresholded & 3758 & 4285 & 5866 & 8501 & 13771 \\
\hline & f-measure thresholded & 3325 & 4165 & 5865 & 7280 & 9615 \\
\hline & f-measure unthresholded & 3567 & 4169 & 5975 & 8985 & 15005 \\
\hline \multirow{4}{*}{ Segment } & AUROC thresholded & 75 & 105 & 195 & 220 & 405 \\
\hline & AUROC unthresholded & 79 & 105 & 183 & 313 & 573 \\
\hline & f-measure thresholded & 50 & 70 & 160 & 220 & 370 \\
\hline & f-measure unthresholded & 61 & 82 & 145 & 250 & 460 \\
\hline \multirow{4}{*}{ E-State } & AUROC thresholded & 12985 & 16510 & 20590 & 22920 & 24085 \\
\hline & AUROC unthresholded & 12985 & 14095 & 17425 & 22975 & 34075 \\
\hline & f-measure thresholded & 11765 & 15930 & 20655 & 22960 & 23645 \\
\hline & f-measure unthresholded & 11765 & 13215 & 17565 & 24815 & 39315 \\
\hline \multirow{4}{*}{ Page } & AUROC thresholded & 945 & 1305 & 1860 & 2315 & 3370 \\
\hline & AUROC unthresholded & 1055 & 1259 & 1871 & 2891 & 4931 \\
\hline & f-measure thresholded & 865 & 1260 & 1750 & 2265 & 2915 \\
\hline & f-measure unthresholded & 976 & 1224 & 1968 & 3208 & 5688 \\
\hline \multirow{4}{*}{ Satimage } & AUROC thresholded & 2330 & 3505 & 5880 & 7580 & 9705 \\
\hline & AUROC unthresholded & 2763 & 3679 & 6427 & 11007 & 20167 \\
\hline & f-measure thresholded & 2480 & 3815 & 5970 & 7675 & 9805 \\
\hline & f-measure unthresholded & 2902 & 3852 & 6702 & 11452 & 20952 \\
\hline \multirow{4}{*}{ Compu. } & AUROC thresholded & 1330 & 2285 & 4075 & 4770 & 6025 \\
\hline & AUROC unthresholded & 1330 & 1690 & 2770 & 4570 & 8170 \\
\hline & f-measure thresholded & 1200 & 1950 & 3815 & 4680 & 6505 \\
\hline & f-measure unthresholded & 990 & 1375 & 2530 & 4455 & 8305 \\
\hline \multirow{4}{*}{ Oil } & AUROC thresholded & 220 & 405 & 710 & 1080 & 1630 \\
\hline & AUROC unthresholded & 290 & 387 & 678 & 1163 & 2133 \\
\hline & f-measure thresholded & 215 & 370 & 770 & 1140 & 1560 \\
\hline & f-measure unthresholded & 277 & 383 & 701 & 1231 & 2291 \\
\hline \multirow{4}{*}{ Mammo. } & AUROC thresholded & 1200 & 1895 & 3465 & 4940 & 7840 \\
\hline & AUROC unthresholded & 1451 & 1805 & 2867 & 4637 & 8177 \\
\hline & f-measure thresholded & 925 & 1550 & 2860 & 4205 & 6835 \\
\hline & f-measure unthresholded & 1165 & 1528 & 2617 & 4432 & 8062 \\
\hline
\end{tabular}

\section{Appendix A. Cost Comparison between between Thresholded and Unthresholded C4.5 leaf estimates after smoothing.}

The above table contains experimental results comparing costs between $\mathrm{C} 4.5$ decision trees learned using the wrapper-AUROC and wrapper-fmeasure. The classifiers were learned in the same manner; however, they differ in the method of evaluation on unseen examples. Unthresholded is simply the decision at the leaf based on the 0.5 threshold. Those using thresholded decisions follow the technique outlined in Section 2.6, which use the posterior probabilities and the known cost ratio to generate an optimal decision. We note that thresholded predictions tend to be stronger at higher cost ratios, but weaker in some cases on lower cost ratios. 


\section{References}

N. B. Amor, S. Benferhat, and Z. Elouedi. Naive Bayes vs. Decision Trees in Intrusion Detection Systems. In Proceedings of the ACM Symposium on Applied Computing, pages 420-424, 2004.

R. E. Banfield, L. O. Hall, K. W. Bowyer, and W. P. Kegelmeyer. Ensembles of Classifiers from Spatially Disjoint Data. In Proceedings of the Sixth International Conference on Multiple Classifier Systems, 2005.

G. E. A. P. A. Batista, R. C. Prati, and M. C. Monard. A study of the behavior of several methods for balancing machine learning training data. SIGKDD Explorations, 6(1), 2004.

K. W. Bowyer, L. O. Hall, N. V. Chawla, and T. E. Moore. A parallel Decision Tree Builder for Mining Very Large Visualization Datasets. In Proceedings of the IEEE International Conference on Systems, Man and Cybernetics, 2000.

L. Breiman. Bagging Predictors. Machine Learning, 24(2):123-140, 1996.

N. V. Chawla, K. W. Bowyer, L. O. Hall, and W. P. Kegelmeyer. SMOTE: Synthetic Minority Over-sampling Technique. Journal of Artificial Intelligence Research, 16:321$357,2002$.

N. V. Chawla, N. Japkowicz, and A. Kołcz, editors. Proceedings of the ICML'2003 Workshop on Learning from Imbalanced Data Sets, 2003.

N. V. Chawla, N. Japkowicz, and A. Kolcz, editors. SIGKDD Explorations Special Issue on Learning from Imbalanced Datasets, 2004. SIGKDD.

N. V. Chawla, L. O. Hall, and A. Joshi. Wrapper-based Computation and Evaluation of Sampling Methods for Imbalanced Datasets. In KDD Workshop: Utility-Based Data Mining, 2005.

D. Cieslak and N. V. Chawla. Calibration and power of PETs on unbalanced datasets, 2006.

W. W. Cohen. Fast effective rule induction. In Armand Prieditis and Stuart Russell, editors, 12th International Conference on Machine Learning, pages 115-123, Tahoe City, CA, 1995a. Morgan Kaufmann.

W. W. Cohen. Learning to classify English text with ILP methods. In 5th International Workshop on Inductive Logic Programming, pages 3-24, 1995b.

T. Dietterich, D. Margineantu, F. Provost, and P. Turney, editors. Proceedings of the ICML'2000 Workshop on COST-SENSITIVE LEARNING, 2003.

C.L. Blake D.J. Newman, S. Hettich and C.J. Merz. UCI repository of machine learning databases, 1998. URL http://www.ics.uci.edu/ mlearn/MLRepository.html.

P. Domingos. MetaCost: A General Method for Making Classifiers Cost-Sensitive. In Knowledge Discovery and Data Mining, pages 155-164, 1999. 
C. Drummond and R. Holte. C4.5, class imbalance, and cost sensitivity: Why undersampling beats over-sampling. In Proceedings of the ICML'03 Workshop on Learning from Imbalanced Data Sets, 2003.

C. Drummond and R. C. Holte. Cost curves: An improved method for visualizing classifier performance. Machine Learning, 65(1):95 - 130, 2006.

S. Dumais, J. Platt, D. Heckerman, and M. Sahami. Inductive learning algorithms and representations for text categorization. In Seventh International Conference on Information and Knowledge Management, pages 148-155, 1998.

C. Elkan. The foundations of cost-sensitive learning. In Proceedings of the Seventeenth International Joint Conference on Artificial Intelligence, pages 973-978, 2001.

C. Elkan. Results of the KDD'99 Classifier Learning Contest. http://www.cse.ucsd.edu/ elkan/clresults.html, 1999.

F. Esposito, D. Malerba, and G. Semeraro. Multistrategy Learning for Document Recognition. Applied Artificial Intelligence, 8:33-84, 1994.

C. Ferri, P. Flach, J. Orallo, and N. Lachice, editors. ECAI'2004 First Workshop on ROC Analysis in AI, 2004. ECAI.

M. Kubat and S. Matwin. Addressing the Curse of Imbalanced Training Sets: One Sided Selection. In Proceedings of the Fourteenth International Conference on Machine Learning, pages 179-186, Nashville, Tennesse, 1997. Morgan Kaufmann.

M. Kubat, R. Holte, and S. Matwin. Machine Learning for the Detection of Oil Spills in Satellite Radar Images. Machine Learning, 30:195-215, 1998a.

M. Kubat, R. C. Holte, and S. Matwin. Machine Learning for the Detection of Oil Spills in Satellite Radar Images. Machine Learning, 30(2-3):195-215, 1998b.

D. Lewis and M. Ringuette. A comparison of two learning algorithms for text categorization. In 3rd Annual Symposium on Document Analysis and Information Retrieval, pages 81-93, 1994 .

C. Ling and C. Li. Data Mining for Direct Marketing Problems and Solutions. In Proceedings of the Fourth International Conference on Knowledge Discovery and Data Mining (KDD98), New York, NY, 1998. AAAI Press.

M. Maloof. Learning when data sets are imbalanced and when costs are unequal and unknown. In Proceedings of the ICML'03 Workshop on Learning from Imbalanced Data Sets, 2003.

D. Mladenic and M. Grobelnik. Feature selection for unbalanced class distribution and naive bayes. In ICML, 1999.

F. J. Provost and P. Domingos. Tree induction for probability-based ranking. Machine Learning, 52(3), 2003. 
F. J. Provost, T. Fawcett, and R. Kohavi. The Case Against Accuracy Estimation for Comparing Induction Algorithms. In Fifteenth International Conference on Machine Learning, pages 445-453, 1998.

J. R. Quinlan. Programs for Machine Learning. Morgan Kaufmann, 1993.

M. R. Sabhnani and G. Serpen. Application of Machine Learning Algorithms to KDD Intrusion Detection Dataset with Misuse Detection Context. In Proceedings of the International Conference on Machine Learning: Models, Technologies, and Applications, pages 209-215, 2003.

D. Thain, T. Tannenbaum, and M. Livny. Distributed computing in practice: The condor experience. Concurrency and Computation: Practice and Experience, 17:323 - 356, 2005.

G. Weiss and F. Provost. Learning when Training Data are Costly: The Effect of Class Distribution on Tree Induction. Journal of Artificial Intelligence Research, 19:315-354, 2003.

Gar Weiss, Kate McCarthy, and Bibi Zabar. Cost-Sensitive Learning vs. Sampling: Which is Best for Handling Unbalanced Classes with Unequal Error Costs? In ICDM, pages 35-41, 2007.

I. H. Witten and E. Frank. Data Mining: Practical machine learning tools and techniques. Morgan Kaufmann, 2005.

K. Woods, C. Doss, K. W. Bowyer, J. Solka, C. Priebe, and W. P. Kegelmeyer. Comparative Evaluation of Pattern Recognition Techniques for Detection of Microcalcifications in Mammography. Internation Journal of Pattern Recognition and Artificial Intelligence, 7 (6), 1993.

B. Zadrozny and C. Elkan. Learning and Making Decisions When Costs and Probabilities are Both Unknown. In Proceedings of the Sixth ACM SIGKDD International Conference on Knowledge Discovery and Data Mining, pages 204-213, 2001.

B. Zadrozny, J. Langford, and N. Abe. A simple method for cost-sensitive learning, 2003a.

B. Zadrozny, J. Langford, and A. Naoki. Cost-Sensitive Learning by Cost- Proportionate Example Weighting. In 2003 IEEE International Conference on Data Mining (ICDM'03), 2003b.

Z. Zhou and X. Liu. Training cost-sensitive neural networks with methods addressing the class imbalance problem. IEEE Transactiosn on Knowledge and Data Engineering, 18 (1):63-77, 2006. 